\title{
Gut metabolomics profiling of non-small cell lung cancer (NSCLC) patients under immunotherapy treatment
}

\author{
Andrea Botticelli ${ }^{1,2+}$, Pamela Vernocchi ${ }^{3 \dagger}$, Federico Marini $i^{4,5}$, Andrea Quagliariello ${ }^{3}$, Bruna Cerbelli ${ }^{6}$, \\ Sofia Reddel ${ }^{3}$, Federica Del Chierico ${ }^{3}$, Francesca Di Pietro ${ }^{7}$, Raffaele Giusti ${ }^{7}$, Alberta Tomassini ${ }^{4,5}$, \\ Ottavia Giampaolii, Alfredo Miccheli ${ }^{5,8}$, Ilaria Grazia Zizzari ${ }^{9}$, Marianna Nuti ${ }^{9}$, Lorenza Putignani ${ }^{10^{*}+}$ (1) \\ and Paolo Marchetti $1,2,7+$
}

\begin{abstract}
Background: Despite the efficacy of immune checkpoint inhibitors (ICls) only the $20-30 \%$ of treated patients present long term benefits. The metabolic changes occurring in the gut microbiota metabolome are herein proposed as a factor potentially influencing the response to immunotherapy.

Methods: The metabolomic profiling of gut microbiota was characterized in 11 patients affected by non-small cell lung cancer (NSCLC) treated with nivolumab in second-line treatment with anti-PD-1 nivolumab. The metabolomics analyses were performed by GC-MS/SPME and ${ }^{1} \mathrm{H}-\mathrm{NMR}$ in order to detect volatile and non-volatile metabolites. Metabolomic data were processed by statistical profiling and chemometric analyses.

Results: Four out of 11 patients (36\%) presented early progression, while the remaining 7 out of 11 (64\%) presented disease progression after 12 months. 2-Pentanone (ketone) and tridecane (alkane) were significantly associated with early progression, and on the contrary short chain fatty acids (SCFAs) (i.e., propionate, butyrate), lysine and nicotinic acid were significantly associated with long-term beneficial effects.

Conclusions: Our preliminary data suggest a significant role of gut microbiota metabolic pathways in affecting response to immunotherapy. The metabolic approach could be a promising strategy to contribute to the personalized management of cancer patients by the identification of microbiota-linked "indicators" of early progressor and long responder patients.
\end{abstract}

\section{Background}

Despite the efficacy of immune checkpoint inhibitors (ICIs) only $20-30 \%$ of treated patients present long term advantages [1].

\footnotetext{
*Correspondence: lorenza.putignani@opbg.net

${ }^{\dagger}$ Andrea Botticelli and Pamela Vernocchi contributed equally to the article

†Lorenza Putignani and Paolo Marchetti are joint senior authors on this work

${ }^{10}$ Unit of Parasitology and Unit of Human Microbiome, Bambino Gesù Children's Hospital, IRCCS, Rome, Italy

Full list of author information is available at the end of the article
}

Although immunohistochemistry detection of programmed death-ligand 1 (PD-L1) has been proposed as a predictive factor in both treatment of naïve and refractory non-small cell lung cancer (NSCLC) patients receiving pembrolizumab, atezolizumab or nivolumab, it presents several limitations such as expression dynamics, different antibody clones used and sampling choice [2].

Recently, tumor mutational burden (TMB) has emerged as an independent biomarker of ICIs outcomes across multiple cancer types, including NSCLC. Carbone et al. [1] recently showed that high TMB, calculated by whole exome sequencing (WES), is

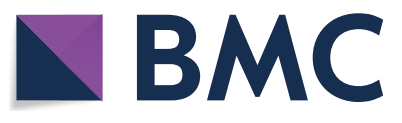

(c) The Author(s) 2020. This article is licensed under a Creative Commons Attribution 4.0 International License, which permits use, sharing, adaptation, distribution and reproduction in any medium or format, as long as you give appropriate credit to the original author(s) and the source, provide a link to the Creative Commons licence, and indicate if changes were made. The images or other third party material in this article are included in the article's Creative Commons licence, unless indicated otherwise in a credit line to the material. If material is not included in the article's Creative Commons licence and your intended use is not permitted by statutory regulation or exceeds the permitted use, you will need to obtain permission directly from the copyright holder. To view a copy of this licence, visit http://creativeco mmons.org/licenses/by/4.0/. The Creative Commons Public Domain Dedication waiver (http://creativecommons.org/publicdomain/ zero/1.0/) applies to the data made available in this article, unless otherwise stated in a credit line to the data. 
associated with improved overall response rate (ORR) ( $46.8 \%$ vs. $28.3 \%$ ) and median progression-free survival (mPFS) [9.7 vs. 5.8 months; hazard ratio for disease progression or death (HR): 0.62; 95\% confidence interval (CI), 0.38 to 1.00$]$ in a group of untreated advanced NSCLC receiving nivolumab compared to platinum chemotherapy treated patients (Checkmate 026 Clinical Trial) [3], and it was proposed as biomarker in the Checkmate 227 study (ClinicalTrials.gov number, NCT02477826) [4]. However, TMB presented some limitation consisting for example in the cut-off score and in the technique adopted i.e., WES or Comprehensive Genomic Profiling (CGP). Furthermore, additional biomarkers are under investigations such as microsatellites [5], interferon signatures [6], T cell repertoire [7], major histocompatibility complex (MHC) status [8], and immune infiltrates [9].

In the context of multiple available biomarkers the changes occurring in the microbiota composition and metabolism have been proposed as a mechanism potentially affecting the response and the toxicity to immunotherapy [10].

Recently, several studies have confirmed the relevant role of the gut microbiota in the modulation of immune functions and its correlation with several diseases, including cancer [11]. In particular, gut-brain and gutliver axes have already been investigated, while the relation between gut-lung axis has been newly suggested [12], in particular the hypothesis that changes in the gut microbiota could influence the lung microbiota, and vice versa (cross talk among microbial communities). However, the local and systemic influence of the gut microbiota, the influence on the lung microbiota and its products have not yet been fully assessed [13]. In fact, little is known about the hypothetical connection when looking at the world's number one cause of death from cancerlung cancer [14].

To date, the abundance of Bifidobacterium species (i.e., cocktail including Bifidobacterium breve and Bifidobacterium longum), has been observed to increase anti-tumor immunity and facilitate anti PD-L1 activity in germ free mice; moreover antibiotic treated mice presented impaired response to anti-Cytotoxic T-Lymphocyte Antigen 4 (CTLA-4) [15].

In melanoma patients treated with antiCTLA4 the presence of Bacteroides seems to have a protective role in terms of gastrointestinal toxicity [16].

Recently, it was also demonstrated that melanoma patients carrying gut microbiota enriched in Faecalibacterium prausnitzii presented longer progression free survival (IPFS) and overall survival (OS) [17], while in patients affected by NSCLC and renal cell carcinoma (RCC) patients treated with anti-PD-1 an higher distribution of Akkermansia muciniphila were assessed in responders compared with non-responders [18].

Despite promising results, the characterization of gut microbiota lacks full functional information about the relationship between the host-diet-microbiota axis, for this reason the study of metabolic profile of microbiota may actually provide new insights to overcome this gap [19].

Hence, as an effort to fill the knowledge gap, metabolomics enclose the comprehensive and concurrent systematic profiling of metabolic changes that occur in living systems in response to set of different factors as pathological, environmental or lifestyle conditions [20]. Metabolomics corroborate and enhances the information provided by genomics and proteomics [21] and have already shown promise in identifying metabolic phenotypes [22, 23].

In the present study, we aimed to characterize the metabolomic profile of NSCLC patients treated with nivolumab [2] and to investigate, for the first time, whether the gut microbiota metabolome may predict a baseline response to immunotherapy.

\section{Materials and methods}

\section{Patient characteristics and sample collection}

From April 2016 to March 2017 a cohort of 11 NSCLC patients aged 44 to 82 years (median age 68 years; 8 males and 3 females) were recruited at the Department of Clinical and Molecular Medicine, Sant' Andrea Hospital, Sapienza University of Rome.

As inclusion criteria the following were considered: adult subjects, age $>18$ years; NSCLC diagnosed by histology; Eastern Cooperative Oncology Group (ECOG) performance status $\leq 2$; anti-PD-1 nivolumab employed as second-line treatment; acceptable pulmonary, cardiac, liver renal, and bone marrow functions.

As exclusion criteria the following were considered: autoimmune diseases; symptomatic interstitial lung disease and other noteworthy comorbidities; systemic immunosuppression; previous treatment with immunestimulatory antitumor agents, including checkpoint-targeted molecules.

Nivolumab was proven at ordinary dosage of $3 \mathrm{mg} / \mathrm{kg}$ each 2 weeks till disease progression or undesirable toxicity. Radiological response was assessed with Response Evaluation Criteria in Solid Tumors (i-RECIST) Criteria and classified according to disease control (complete response, partial response and stable disease) and progressive disease. Toxicity was reported consistently with National Cancer Institute Common Terminology Criteria for Adverse Events (version 4.0) and toxicity valuation was performed at day 1 of every cycle until end of cure. The PFS was defined as the time from 
patient registration on clinical trial until the first documented tumor progression or death from any cause. The OS was defined as the time from patient registration to death from any cause. We defined as early progressors (EPs), patients experiencing disease progression within 3 months from the beginning of nivolumab, and long responders (LRs) patients presenting PFS longer than 12 months. The study was conducted according to good clinical practice guidelines and Helsinki declaration. The final version of the protocol was approved by the Institutional Ethics Committee (Ethical Committee n 4421, "Sapienza University"). Patients gave informed consent according to the guidance of the hospital ethics committee and with the approval of regulatory agencies. The fecal samples collected from NSCLC patients for gut microbiota metabolome profiling, were handled and processed for biobanking and integration processing at the OPBG Human Microbiome Unit and NMRbased Metabolomics Laboratory, Sapienza University of Rome.

\section{Targeted metagenomic on faecal microbiota}

Genomic DNA from stool samples was manually extracted using QIAmp Fast DNA Stool mini kit (Qiagen, Germany), according to the manufacturer's instructions.

Amplification of the variable region V3-V4 from the $16 \mathrm{~S}$ rRNA gene $(\sim 460 \mathrm{bp})$ was carried out using the primer pairs described in the MiSeq rRNA Amplicon Sequencing protocol (Illumina, San Diego, CA).

The so obtained DNA amplicons were then cleanedup by AMPure XP beads (Beckman Coulter Inc., Beverly, MA, USA). After second amplification step using a unique combination of bar-coded Illumina Nextera forward and reverse adaptor-primers, the final library was cleaned-up using $50 \mu \mathrm{L}$ of AMPure XP beads and quantified using Quant-iT ${ }^{\mathrm{TM}}$ PicoGreen ${ }^{\circledR}$ dsDNA Assay Kit (Thermo Fisher Scientific, Waltham, MA). Finally, the library was diluted in equimolar concentrations (4 nM), pooled together and sequenced on an Illumina MiSeqTM platform according to the manufacturer's specifications.

Paired-ends reads were trimmed for their quality, read length and chimera presence using Qiime v1.8. pipeline [24]. Sequences were organized into Operational Taxonomic Units (OTUs) with a 97\% of clustering threshold of pairwise identity and representative sequences were aligned using PyNAST v.0.1. software [25] against Greengenes 13_08 database [26] with a 97\% threshold of similarity. Ecological and statistical analyses were performed using phyloseq and DESeq 2 packages from R software $[27,28]$.

\section{Gut microbiota metabolomics profiling}

Generation of volatilome by gas chromatography solid-phase microextraction (GC-MS/SPME)

Fecal volatile organic compounds (VOCs) from 11 NSCLC patients were detected according to Vernocchi et al. [29] by using the carboxen-polydimethylsiloxane coated fiber (CAR-PDMS) $(85 \mu \mathrm{m})$ and the manual SPME holder (Supelco Inc., Bellefonte, PA, USA) according to Vernocchi et al. [29]. The SPME fiber was exposed to each sample for $45 \mathrm{~min}$. Both phases of equilibration and absorption were carried out under stirring condition. The fiber was then inserted into the GC injection port (10 min) for sample desorption and the GC-MS analyses carried out on an Agilent Technologies 7890B GC, coupled to a 5977A mass selective detector operating in electron impact mode (ionization voltage $70 \mathrm{eV}$ ), within a 1 -mm quartz liner fitted system, equipped with a Supelcowax 10 capillary column $(60 \mathrm{~m}$ length, $0.32 \mathrm{~mm}$ ID, Supelco, Bellefonte, PA, USA). The temperature program was the following: $50{ }^{\circ} \mathrm{C}$ for $1 \mathrm{~min}, 4.5{ }^{\circ} \mathrm{C} / \mathrm{min}$ to $65{ }^{\circ} \mathrm{C}$ and $10{ }^{\circ} \mathrm{C} / \mathrm{min}$ to $230{ }^{\circ} \mathrm{C}$, which was held for $15 \mathrm{~min}$. Injector, interface and ion source temperatures were 250, 250 and $260^{\circ} \mathrm{C}$. Total run time $35.83 \mathrm{~min}$. The mass-scan range was 30-300 a.m.u. at 5.19 scans/s. Injections were carried out in splitless mode, under helium $(1.5 \mathrm{~mL} / \mathrm{min})$ carrier. Molecule identification (ID) was carried out by using retention times (Rt) compared to pure compounds Rt (Sigma-Aldrich, Milan, Italy). The chromatograms were integrated and identified by comparing the fragment pattern with those in the mass spectral NIST library (version 2.2, NIST 14MS database; National Institute of Standards and Technology, Rockville, MD) and literature [30] followed by manual visual inspection. Quantitative compound data were expressed as ppm $(\mathrm{mg} / \mathrm{kg})$ obtained by interpolation of the relative areas vs. IS area.

\section{Determination of non-volatile metabolites by ${ }^{1} \mathrm{H}-\mathrm{NMR}$ spectroscopy}

Fecal water was obtained as previously described [31]. After sample collection, 2 out of 11 NSCLC samples were excluded for insufficient sample collection. Briefly, ${ }^{1} \mathrm{H}$ NMR spectroscopy analysis was performed on $500 \mathrm{mg}$ of feces suspended with $1 \mathrm{~mL}$ of $\mathrm{D}_{2} \mathrm{O}-\mathrm{PBS}-\mathrm{NaN}_{3}$ buffered solution. Each sample was vortexed for $2 \mathrm{~min}$ and then centrifuged for $25 \mathrm{~min}$ at $10,000 \mathrm{rpm}$ and $4{ }^{\circ} \mathrm{C}$ to obtain fecal water. In total, $600 \mu \mathrm{L}$ of supernatant was collected and analyzed according to Brasili et al. [31] NMR analysis was carried out at $298 \mathrm{~K}$ by using a Bruker Advance 400 spectroscope (Bruker BioSpin GmbH, Rheinstetten, Germany), equipped with a magnet operating at 9.4 Tesla (400.13 MHz for ${ }^{1} \mathrm{H}$ frequency). 1D ${ }^{1} \mathrm{H}$ NMR experiment was performed employing the standard presaturation 
presat pulse sequence. Spectral width was set to $6009 \mathrm{~Hz}$ (15 ppm) and 64 scans were collected for each spectrum with a presaturation pulse length of $2.00 \mathrm{~s}$ and a relaxation delay of $6.55 \mathrm{~s}$. The spectra were collected with $64 \mathrm{~K}$ points for an acquisition time of $5.5 \mathrm{~s}$ [32]. The assignment of resonances was done by $2 \mathrm{D}$ homonuclear NMR Total Correlated Spectroscopy (TOCSY) and heteronuclear Single Quantum Coherence (HSQC) experiments. TOCSY experiments were recorded at $298 \mathrm{~K}$ with a spectral width of $15 \mathrm{ppm}$ in both dimensions, using $8 \mathrm{~K} \times 256$ data points matrix, repetition time of $2 \mathrm{~s}$ and 80 scans with a mixing time of $110 \mathrm{~ms}$. HSQC experiments were acquired with a spectral width of $12 \mathrm{ppm}$ in proton dimension and $200 \mathrm{ppm}$ in the carbon dimension, using $8 \mathrm{~K} \times 256$ data points matrix for the proton and the carbon dimensions, respectively, with a repetition delay of $2 \mathrm{~s}$ and 96 scans. The assignment was confirmed according to Human Metabolome Data Base [33] and own laboratory database. $1 \mathrm{D}{ }^{1} \mathrm{H}$ NMR spectra were processed and quantified by using the ACD Lab 1D-NMR Manager 12.0 software (Advanced Chemistry Development, Inc., Toronto, ON, Canada), whereas the MestReC software (Mestrelab Research SL, Santiago de Compostela, Spain) was used to assess 2D-NMR spectra. The quantification of metabolites was obtained by comparison of the integrals (normalized for number of protons) of specific signals with the IS trimethyl silyl propanoic acid (TSP) integral and then normalized for feces weight (expressed as $\mu \mathrm{mol} / \mathrm{g}$ ).

\section{Statistical analysis}

To characterize the differences between NSCLC subjects from a single- and a multi-omic standpoint (i.e., ${ }^{1} \mathrm{H}-\mathrm{NMR}$ - and GC-MS-based metabolomics), a data analytical strategy based on the use of multivariate chemometric methods were carried out on the integrated dataset of 9 patients considering both GC-MS and ${ }^{1} \mathrm{H}$ NMR data. However, in order to have reliable results, prior to data processing, the raw data matrix of GC-MS/ SPME metabolites was cleaned by retaining only those molecules which were detected in at least $80 \%$ of the investigated subjects. Such a screening was not needed in the case of ${ }^{1} \mathrm{H}$-NMR data. Since the two blocks of data came from different experimental techniques and had different variances and because of high inherent variability, data were block scaled after individual autoscaling. Metabolomics multivariate data analysis was carried out using in house written functions running under MATLAB (R2015b; The Mathworks, Natick, MA) environment. Principal components analysis (PCA) was used to analyze inherent clustering, to identify outliers and significant metabolites. Mann-Whitney $U$ test was then applied to assign significant differences at the level of single metabolite in the PCA model in particular, a $p$-value of 0.05 was considered as threshold for statistical significance.

\section{Results}

\section{Clinical characteristics}

Eleven patients with stage IV NSCLC treated with second-line nivolumab were enrolled in this study. Baseline clinical characteristics of patients are summarized in Table 1. Among them, 10 patients had squamous-cell carcinoma and one adenocarcinoma. Median PFS and OS were 7.5 and 7.7 months, respectively. Four patients were EPs, while 7 LRs (Table 1).

\section{Gut microbiota ecological analysis}

The metagenomics data have been analyzed in a preliminary way and no cluster formation has been highlighted between EPs and LRs in the structure of microbiota (Additional file 1: Figure S1).

\section{Table 1 Clinical features of NSCLC patients: phenomic} metadata

\begin{tabular}{|c|c|}
\hline Patient characteristics at baseline & $\mathrm{N}^{\circ}(\%)$ \\
\hline \multicolumn{2}{|l|}{ Age } \\
\hline$\leq 65$ & $5(45)$ \\
\hline$>65$ & $6(55)$ \\
\hline \multicolumn{2}{|l|}{ Sex } \\
\hline Male & $8(73)$ \\
\hline Female & $3(27)$ \\
\hline \multicolumn{2}{|l|}{ Histology } \\
\hline Adenocarcinoma & $1(10)$ \\
\hline Squamous cell carcinoma & $10(90)$ \\
\hline \multicolumn{2}{|l|}{ ECOG performance status } \\
\hline $0-1$ & $10(90)$ \\
\hline$>1$ & $1(10)$ \\
\hline \multicolumn{2}{|l|}{$\mathrm{N}^{0}$ sites of metastasis } \\
\hline 1 & $2(18)$ \\
\hline$>1$ & $9(82)$ \\
\hline \multicolumn{2}{|l|}{ Brain metastases } \\
\hline Present & $1(10)$ \\
\hline Absent & $10(90)$ \\
\hline \multicolumn{2}{|l|}{ Treatment line } \\
\hline 2 & $11(100)$ \\
\hline$>2$ & $0(0)$ \\
\hline \multicolumn{2}{|l|}{ Previous platinum based chemotherapy } \\
\hline Yes & $9(82)$ \\
\hline No & $2(18)$ \\
\hline \multicolumn{2}{|l|}{ Response to nivolumab } \\
\hline Early progressors (EPs) & $4(36)$ \\
\hline Long responder (LRs) & $7(64)$ \\
\hline
\end{tabular}


In particular, the analysis performed by Bray-Curtis dissimilarity (Additional file 1: Figure S1, panel A) does not highlight the existence of a specific cluster related to the clinical condition of EP or LR. Furthermore, this is confirmed in the distribution of the main bacterial genera for each sample (Additional file 1: Figure S1, panel B).

\section{Gut microbiota metabolomics}

Each fecal sample was analysed to determine both VOCs and non-volatile metabolites, in an untargeted fashion, thus capturing large numbers of known and uncharacterized metabolites, including those of potential microbial origin.

\section{VOCs profiling}

Two-hundred and twenty-three VOCs for all 11 NSCLC patients were identified and quantified by GC-MS/ SPME, and grouped into the following 18 chemical classes: alcohols (n 44); esters (n 31); aldehydes (n 21); ketones (n 35); alkenes (n 12); alkanes (n 17); acids (n 8); phenols (n 4); terpenes (n 18); sulfur compounds (n 2); hydrazine (n 1); azetidine (n 1); indoles (n 7); pyridine (n 1); amines (n 13); furans (n 2); pyrazine (n 2) and aromatic hydrocarbons (n 4) (data not shown). After data reduction, a matrix with 24 metabolites detected in the $80 \%$ of samples was considered for computation (Additional file 2: Table S1).

\section{Non-volatile metabolite profiling}

Fourty-nine non volatile metabolites were detected (i.e., acids, amino acids, amines, and sugars) and quantified ( $\mu \mathrm{mol} / \mathrm{g}$ ) by ${ }^{1} \mathrm{H}-\mathrm{NMR}$ for 9 NSCLC patients (Additional file 3: Table S2).

\section{Integrated metabolomic model}

In order to have a multi-omics overview, the integration of GC-MS/SPME and ${ }^{1} \mathrm{H}-\mathrm{NMR}$ data was carried out through a low-level data fusion approach. Therefore, PCA was applied to the matrix obtained by concatenation of GC-MS/SPME and ${ }^{1} \mathrm{H}-\mathrm{NMR}$ data after block-scaling. The PCA scores plot (PC1 vs. PC2; PC1: 28.82\%; PC2: 17.80\%), displayed in Fig. 1, panel a, showed a clear separation between EP and LR patients (Fig. 1, panel a). In particular, the separation between the two groups occurred mainly along the axis of PC1; therefore, in order to assess which metabolites mainly contributes to the observed separation, only the variables having the highest absolute value of the loadings on such component were considered (Fig. 1, panel b).

Accordingly, inspection of the loadings plot allowed observing how the gut microbiota metabolome of LRs patients was mostly characterized by SCFAs (i.e., butyric, valeric, acetic and propionic), AAs (i.e., lysine) and nicotinic acid. On the contrary, EPs were mainly represented by alcanes (i.e., tridecane, dodecane) ketones (i.e., 2-pentanone, 2-octanone), aldehydes (i.e., benzeneacetaldehyde), and $p$-cresol. For glutamic acid, isoleucine, 2-octanone, valeric acid, acetic acid, butyric acid and dodecane the level differences between EPs and LRs subjects were not statistically significant (data not shown).

Interestingly, the levels of tridecane could be measured only in fecal metabolome of EPs patients, being absent in that of LR ones. Particularly, the differences between levels of propionic acid ( $p$ value $=0.016$ ), lysine ( $\mathrm{p}$ value $=0.032)$, nicotinic acid ( $\mathrm{p}$ value $=0.016$ ), tridecane $(\mathrm{p}$ value $=0.032) 2$-pentanone $(\mathrm{p}$ value $=0.016)$ and $p$-cresol ( $\mathrm{p}$ value $=0.016$ ) were statistically significant, as inferred by U-Mann-Whitney test. PCA model took into account the covariance of propionic acid with the other SCFAs (Fig. 2).

\section{Discussion}

Some evidences suggest that gut microbiota-induced immune effects dependent on the specific therapy for different type of cancer [15, 34, 35].

Chemotherapy could impact on both immune system and gut microbiota, influencing the relationship between both of them [14].

By metagenomics approach no statistical significant association were found between EPs and LRs; moreover

\footnotetext{
(See figure on next page.)

Fig. 1 Principal component analysis (PCA) of integrated datasets of ${ }^{1} \mathrm{H}-\mathrm{NMR}$ and GC-MS/SPME data at $\mathrm{T}_{0}$. a PC score plot. $\mathbf{b}$ Loading plot. The first two components explained $47 \%$ of the total variance. In green and red circles are represented EP (early progressors, not responders) and LR (long responders) patients, respectively. a Red, early progressors (EPS); green, long responders (LRs). b 1: 1-butanol; 2: 1-hexanol; 3: 1-pentanol; 4: 2,6-dimethyl 4 heptanone; 5: 2-butanone; 6: 2-heptanone; 7: 2-hexanol; 8: 2-nonanone; 9: 2-octanol; 10: 2-octanone; 11: 2-pentanone; 12: 6-methyl-5-hepten-2-one; 13: benzaldehyde; 14: benzeneacetaldehyde; 15: cis-2,6-dimethyl-2,6-octadiene; 16: dimethyl disulfide; 17: dodecane; 18: indole; 19: methyl isobutyl ketone; 20: p-cresol; 21: tridecane; 22: Bile salt 1; 23: Bile salt 2; 24: U1; 25: 2-hydroxy-3-methylbutyric acid 26: U2; 27: valeric acid; 28: isovaleric acid; 29: leucine; 30: valine; 31: isoleucine; 32: U3; 33: 2-oxoisovaleric acid; 34: ethanol; 35: lactic acid; 36: acetoin; 37 : 2-aminoisobutyrate; 38: alanine; 39: butyric acid; 40: Iysine 41: acetic acid; 42: N-acetyl-moieties; 43: propionic acid; 44: glutamic acid; 45: succinic acid; 46: U4; 47: methionine; 48: aspartic acid; 49: trimethylamine (TMA); 50: 2-oxoglutarate; 51: malonic acid; 52: U5; 53: choline; 54: taurine; 55: methanol; 56: glycine; 57: b-arabinose; 58: b-galactose; 59: b-xylose; 60: b-glucose; 61: U6; 62: uracil; 63: orotic acid; 64: U7; 65: fumaric acid; 66: tyrosine; 67: phenylalanine; 68: U8; 69: formic acid; 70: nicotinic acid
} 

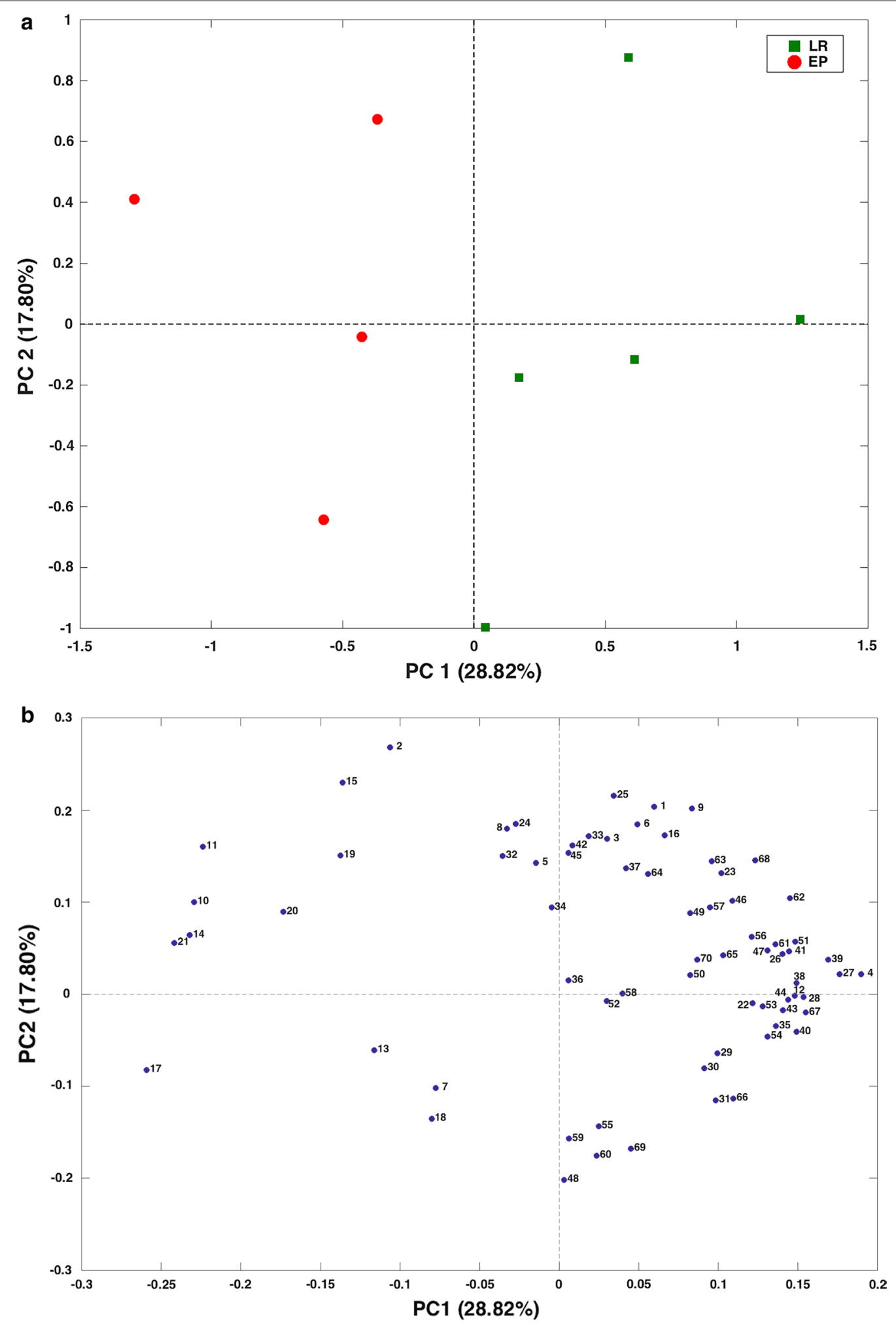

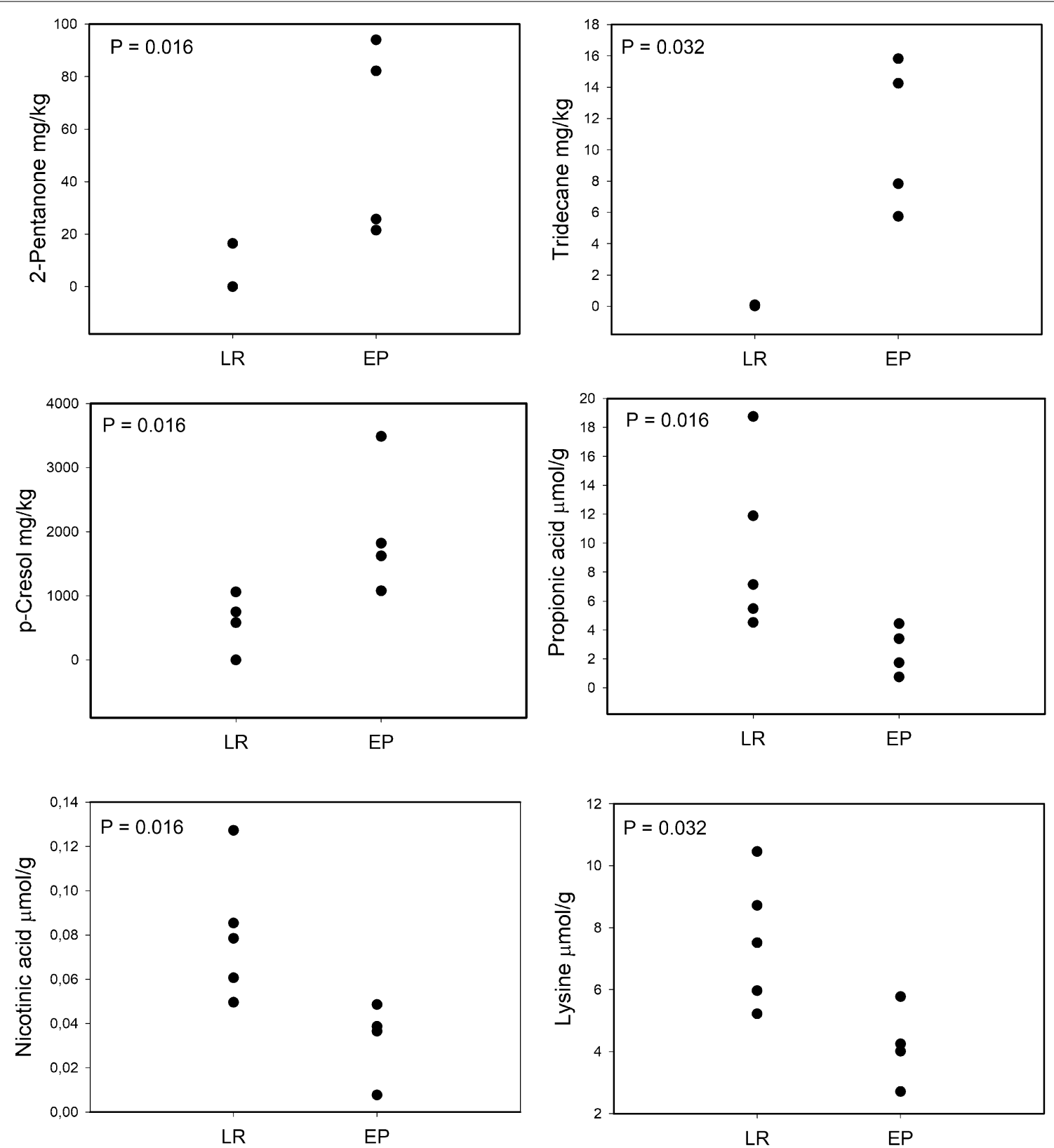

Fig. 2 Concentration ( $\mu \mathrm{mol} / \mathrm{g}$ ) of proprionic acid (median $L R=7.14 \mathrm{EP}=2.56$ ), nicotinic acid (median $L R=0.08 \mathrm{EP}=0.04$ ), lysine (median $L R=7.51$ $E P=4.13)$, 2-pentanone (median $L R=0 ; E P=53.9)$ tridecane (median $L R=0 ; E P=11.03$ ) and $p$-cresol (median $L R=582.91 ; E P=1721.48$ )

it was not possible to hypothesize a potential relationship with the clinical outcomes.

Untargeted metabolomics may contribute to highlight gut microbiota metabolites allowing to infer hostmicrobiome co-metabolism [36]. The application of multivariate chemometric model based on the GC-MS and ${ }^{1} \mathrm{H}$-NMR data may concur to clarify the responsiveness to immune therapy of NSCLC patients under specific disease conditions.
The gut microbiota metabolome in cancer has been particularly investigated to give information on the relationship between colorectal cancer and gut microbiome. However, the role of specific bacteria metabolism in carcinogenesis and progression remains an active area of inquiry [37].

Metabolites and VOCs have been investigated in lung cancer on exhaled breath [38-40] and urine [41, 42]. Therefore, low weight molecules have been proposed 
as biomarkers of lung cancer. Up to date the interindividual variations of gut microbiota composition have been studied to find a possible relationship between enterotype and immune-checkpoint therapy response in NSCLC patients $[14,18]$. In the present study, by using a metabolomics approach, was detected for the first time a gut microbiota metabolome potentially associated to NSCLC patients responsiveness to immune checkpoint inhibitors (ICIs). Fecal samples of EPs NSCLC patients were characterized by a metabolic profile constituted by high levels of alkanes (tridecane and dodecane), methylketones (2-pentanone and 2-octanone), $p$-cresol, and low levels of SCFAs (propionic, butyric, acetic, valeric acids), aminoacids (lysine, isoleucine and glutamic) and nicotinic acid suggesting an imbalanced microbiota metabolism in agreement with a dysbiotic intestinal microbiota as reported in previous studies [18]. Patients affected by NSCLC with longer progressive free-survival have an higher abundance of Akkermansia muciniphila and of other commensales such as Ruminococcus spp., Alistipes spp., and Eubacterium spp., with a relative under representation of Bifidobacterium adolescentis, B. longum, and Parabacteroides distasonis [18]. Akkermansia muciniphila is a mucin degrader producing propionate through propanediol pathway, as well as some of Ruminococcus spp. fermenting fucose, released from mucin degradation, for the propionate production [43]. Generally, SCFAs reach very high concentrations in the colon [44], reducing $\mathrm{pH}$, satisfying nutritional requirements, microbial function regulation and composition, and also affecting the immune system [45]. SCFAs regulate innate immune cells such as neutrophils acting as neutrophil chemotaxin, macrophages and dendritic cells (DCs) by G-protein-joined receptors (GPCRs) and HDACs and also modulate bidirectionally antigen-specific adaptive immunity mediated by T-cells and B-cells.

In fact, SCFAs regulate the immune system by histone deacetylases (HDACs), receptors and/or metabolic integration.

Heerdt et al. [46] highlighted that SCFAs are generally perceived as tumor suppressors as they induce cancer cell differentiation and apoptosis. Acetic and butyric acid levels were reduced in patients with colitis, colon cancer, or other intestinal disorders as inflammatory bowel disorder $[47,48]$.

The gut-lung axis theory [49] suggests that microbes or their metabolic products might have systemic effects, and hence, could give an effect on the lung bacterial composition and immune response. For this reason, fecal SCFAs, which are considered immune modulators/protectors of the intestinal barrier [50], might be possible mediators of the gut "long-distance" affecting directly or indirectly the target site by the stimulation of gut/circulating immune system [14]. Therefore, the gut microbiota of EPs patients appears characterized by a low metabolic activity producing SCFAs associated to an high production of $p$-cresol as compared to LRs. $P$-cresol is a microbial metabolite produced from tyrosine through tyrosine lyase or tyrosine aminotransferase $\beta$ activity; $p$-cresol producers belonged to the Coriobacteriaceae and Clostridium clusters XI and XIVa [51]. The $p$-cresol exhibits cytotoxicity and genotoxicity and reduces endothelial barrier function in vitro $[52,53] . P$-cresyl sulfate, a sulfate-conjugate of $p$-cresol, suppresses Th1-type cellular immune responses in mice $[39,42]$. High levels of this metabolite in urine have been found in patients with cardiovascular and renal diseases [51]. Finally, low levels of nicotinic acid (niacin) complete the metabolic profile of EPs patients. In general, the niacin levels in the feces depend on the food intake, however gut bacteria metabolism could also contribute to the fecal levels of this metabolite [54]. Niacin is a important precursor for the synthesis of nicotinamide adenine dinucleotide (NAD+) that is the central cofactor of metabolism, mediating ATP generation, energy substrate oxidation, reactive oxygen species (ROS) detoxification, DNA repair, and nutritionally sensitive gene regulation [55].

Indeed, in general, metabolome encloses the comprehensive systematic profiling of metabolic changes that occur in living systems in response to sets of different factors such as pathological, environmental or lifestyle conditions [56]. Gut microbiota metabolomic profile corroborates and enhances the information provided by genomics and proteomics [57] and has already shown promises in identifying metabolic phenotypes associated to microbiota patterns [22].

\section{Conclusion}

In our study SCFAs mainly characterize the gut microbiota metabolome of LRs subjects to immune therapy and therefore it can be hypothesized that these molecules might be considered as biomarker of responsiveness. On the contrary, the fecal levels of tridecane might be considered as biomarker of non-responsiveness.

These results should be of support to better understand the close interaction between the gut microbiota of different "communicating" body districts and their interaction with the immune system in NSCLC patients. The power of this study approach is that data collection through non-invasive techniques can be incorporated into standard laboratory exams, hence modulating treatment of the patients.

However, it is necessary to pursue with larger followup clinical studies, in order to provide more representative datasets. The mechanism how these microbial metabolites alone or in combination modulate the host immune system remains to be highlighted. Hence, 
the identification of biomarkers might help in designing new personalized or "alternative" therapies in lung cancer treatment, with also a better characterization of patient's status and diagnosis, as well as in searching new ways to improve immunotherapy tolerance and immune therapies responses, also implemented with nutritional support as pre, pro, postbiotics and symbiotics.

\section{Supplementary information}

Supplementary information accompanies this paper at https://doi. org/10.1186/s12967-020-02231-0.

Additional file 1: Figure S1. Panel A. Analysis of Bray-curtis of the analyzed samples divided by EP (blue circle) and LR (yellow triangle). Panel B. Distribution of the relative abundances of bacterial genera present in addition to $0.01 \%$ for each sample in the two groups considered EP and LR.

Additional file 2: Table S1. GC-MS/SPME metabolites raw data.

Additional file 3: Table S2. ${ }^{1} \mathrm{H}-\mathrm{NMR}$ metabolites raw data.

\section{Acknowledgements}

We wish to dedicate this article in lovely memory of Giuliano, a special person and physician with a great passion for medicine and research who has fighted against the disease with extraordinary dignity and awareness.

\section{Authors' contributions}

$A B$ conceived the study. $A B, L P$ and $P M$ designed the work. $A B, P V, L P, M N$ wrote the manuscript. PV, SR, IGZ, FRD, RG acquired the samples, performed the experiments and acquired the data. FM, PV, FDC, AQ, AT, OG, AM analysed the data. $A B, P V, L P, M N, P M$ discussed results and implications of findings. $B C$ revised English language of the manuscript. All authors read and approved the final manuscript.

\section{Funding}

This work was supported by Ministry of Health through Ricerca Corrente 201802 G004314 assigned to LP and by Sapienza University of Rome.

\section{Availability of data and materials}

All data generated and analyzed during the current study are included in this published article.

\section{Ethics approval and consent to participate}

This study was approved by a local ethics committee RIF. CE: 4421.

\section{Consent for publication}

All the patients provided written informed consent.

\section{Competing interests}

Paolo Marchetti (PM) has/had a consultant/advisory role for BMS, Roche Genentech, MSD, Novartis, Amgen, Merck Serono, Pierre Fabre, Incyte. The other authors declare that they have no competing interests.

\section{Author details}

${ }^{1}$ Department of Clinical and Molecular Medicine, Sapienza University of Rome, Rome, Italy. ${ }^{2}$ AOU Policlinico Umberto I, Rome, Italy. ${ }^{3}$ Unit of Human Microbiome, Bambino Gesù Children's Hospital, IRCCS, Rome, Italy. ${ }^{4}$ Department of Chemistry, Sapienza University of Rome, Rome, Italy. ${ }^{5}$ NMR-based Metabolomics Laboratory, Sapienza University of Rome, Rome, Italy. ${ }^{6}$ Department of Medico-Surgical Sciences and Biotechnologies, Sapienza University of Rome, Rome, Italy. ${ }^{7}$ AOU Sant'Andrea Hospital, Rome, Italy. ${ }^{8}$ Department of Enviromental Biology, University of Rome, Rome, Italy. ${ }^{9}$ Department of Experimental Medicine, University Sapienza, Rome, Italy. ${ }^{10}$ Unit of Parasitology and Unit of Human Microbiome, Bambino Gesù Children's Hospital, IRCCS, Rome, Italy.
Received: 20 August 2019 Accepted: 16 January 2020

Published online: 03 February 2020

\section{References}

1. Darvin P, Toor SM, Sasidharan Nair V, Elkord E. Immune checkpoint inhibitors: recent progress and potential biomarkers. Exp Mol Med. 2018;50:165.

2. Kim H, Chung J-H. PD-L1 testing in non-small cell lung cancer: past, present, and future. J Pathol Transl Med. 2019;53:199-206.

3. Carbone DP, Reck M, Paz-Ares L, Creelan B, Horn L, Steins M, et al. First-line nivolumab in stage IV or recurrent non-small-cell lung cancer. N Engl J Med. 2017;376:2415-26.

4. Hellmann MD, Ciuleanu T-E, Pluzanski A, Lee JS, Otterson GA, AudigierValette $C$, et al. Nivolumab plus ipilimumab in lung cancer with a high tumor mutational burden. N Engl I Med. 2018;378:2093-104.

5. Kato S, Subbiah V, Kurzrock R. Counterpoint: successes in the pursuit of precision medicine: biomarkers take credit. J Natl Compr Cancer Netw. 2017;15:863-6.

6. Ayers M, Lunceford J, Nebozhyn M, Murphy E, Loboda A, Kaufman DR, et al. IFN- - -related mRNA profile predicts clinical response to PD-1 blockade. J Clin Invest. 2017;127:2930-40.

7. Cha E, Klinger M, Hou Y, Cummings C, Ribas A, Faham M, et al. Improved survival with T cell clonotype stability after anti-CTLA-4 treatment in cancer patients. Sci Transl Med. 2014;6:238ra70.

8. Chowell D, Morris LGT, Grigg CM, Weber JK, Samstein RM, Makarov V, et al. Patient HLA class I genotype influences cancer response to checkpoint blockade immunotherapy. Science. 2018;359:582-7.

9. Salgado R, Denkert C, Demaria S, Sirtaine N, Klauschen F, Pruneri G, et al. The evaluation of tumor-infiltrating lymphocytes (TILs) in breast cancer: recommendations by an International TILs Working Group 2014. Ann Oncol. 2015;26:259-71.

10. Fessler J, Matson V, Gajewski TF. Exploring the emerging role of the microbiome in cancer immunotherapy. J Immunother Cancer. 2019;7:108.

11. Rea D, Coppola G, Palma G, Barbieri A, Luciano A, Del Prete P, et al. Microbiota effects on cancer: from risks to therapies. Oncotarget. 2018;9:17915-27.

12. Anand S, Mande SS. Diet, microbiota and gut-lung connection. Front Microbiol. 2018;9:2147.

13. Sze MA, Tsuruta M, Yang S-WJ, Oh Y, Man SFP, Hogg JC, et al. Changes in the bacterial microbiota in gut, blood, and lungs following acute LPS instillation into mice lungs. PLoS ONE. 2014;9:e111228.

14. Bingula R, Filaire M, Radosevic-Robin N, Berthon J-Y, Bernalier-Donadille A, Vasson M-P, et al. Characterisation of gut, lung, and upper airways microbiota in patients with non-small cell lung carcinoma: study protocol for case-control observational trial. Medicine. 2018;97:e13676.

15. Sivan A, Corrales L, Hubert N, Williams JB, Aquino-Michaels K, Earley ZM, et al. Commensal bifidobacterium promotes antitumor immunity and facilitates anti-PD-L1 efficacy. Science. 2015;350:1084-9.

16. Dubin K, Callahan MK, Ren B, Khanin R, Viale A, Ling L, et al. Intestinal microbiome analyses identify melanoma patients at risk for checkpointblockade-induced colitis. Nat Commun. 2016;7:10391.

17. Gopalakrishnan V, Spencer CN, Nezi L, Reuben A, Andrews MC, Karpinets TV, et al. Gut microbiome modulates response to anti-PD-1 immunotherapy in melanoma patients. Science. 2018;359:97-103.

18. Routy B, Le Chatelier E, Derosa L, Duong CPM, Alou MT, Daillère R, et al. Gut microbiome influences efficacy of PD-1-based immunotherapy against epithelial tumors. Science. 2018;359:91-7.

19. Zierer J, Jackson MA, Kastenmüller G, Mangino M, Long T, Telenti A, et al. The fecal metabolome as a functional readout of the gut microbiome. Nat Genet. 2018;50:790-5.

20. Nicholson JK, Holmes E, Kinross J, Burcelin R, Gibson G, Jia W, et al. Hostgut microbiota metabolic interactions. Science. 2012;336:1262-7.

21. Lindon JC, Nicholson JK, Holmes E, editors. The handbook of metabonomics and metabolomics. 1st ed. Amsterdam: Elsevier; 2007.

22. Vernocchi P, Vannini L, Gottardi D, Del Chierico F, Serrazanetti DI, Ndagijimana $\mathrm{M}$, et al. Integration of datasets from different analytical techniques to assess the impact of nutrition on human metabolome. Front Cell Infect Microbiol. 2012;2:156. 
23. Holmes E, Wilson ID, Nicholson JK. Metabolic phenotyping in health and disease. Cell. 2008;134:714-7.

24. Caporaso JG, Kuczynski J, Stombaugh J, Bittinger K, Bushman FD, Costello EK, et al. QIIME allows analysis of high-throughput community sequencing data. Nat Methods. 2010;7:335-6.

25. Caporaso JG, Bittinger K, Bushman FD, DeSantis TZ, Andersen GL, Knight R. PyNAST: a flexible tool for aligning sequences to a template alignment. Bioinformatics. 2010;26:266-7.

26. DeSantis TZ, Hugenholtz P, Larsen N, Rojas M, Brodie EL, Keller K, et al. Greengenes, a chimera-checked 16S rRNA gene database and workbench compatible with ARB. Appl Environ Microbiol. 2006;72:5069-72.

27. McMurdie PJ, Holmes S. phyloseq: an R package for reproducible interactive analysis and graphics of microbiome census data. PLOS ONE. 2013;8:e61217.

28. Love MI, Huber W, Anders S. Moderated estimation of fold change and dispersion for RNA-seq data with DESeq2. Genome Biol. 2014;15:550.

29. Vernocchi P, Del Chierico F, Russo A, Majo F, Rossitto M, Valerio M, et al. Gut microbiota signatures in cystic fibrosis: loss of host CFTR function drives the microbiota enterophenotype. PLOS ONE. 2018;13:e0208171.

30. Garner CE, Smith S, de Lacy Costello B, White P, Spencer R, Probert CSJ, et al. Volatile organic compounds from feces and their potential for diagnosis of gastrointestinal disease. FASEB J. 2007;21:1675-88.

31. Brasili E, Hassimotto NMA, Del Chierico F, Marini F, Quagliariello A, Sciubba F, et al. Daily consumption of orange juice from Citrus sinensis L. Osbeck cv. Cara Cara and cv. Bahia differently affects gut microbiota profiling as unveiled by an integrated meta-omics approach. J Agric Food Chem. 2019;67:1381-91.

32. Brasili E, Mengheri E, Tomassini A, Capuani G, Roselli M, Finamore A, et al Lactobacillus acidophilus La5 and Bifidobacterium lactis Bb12 induce different age-related metabolic profiles revealed by $1 \mathrm{H}-\mathrm{NMR}$ spectroscopy in urine and feces of mice. J Nutr. 2013:143:1549-57.

33. Wishart DS, Jewison T, Guo AC, Wilson M, Knox C, Liu Y, et al. HMDB 30-the human metabolome database in 2013. Nucleic Acids Res. 2013;41:D801-7.

34. Vétizou M, Pitt JM, Daillère R, Lepage P, Waldschmitt N, Flament $C$, et al. Anticancer immunotherapy by CTLA-4 blockade relies on the gut microbiota. Science. 2015;350:1079-84.

35. Frankel AE, Coughlin LA, Kim J, Froehlich TW, Xie Y, Frenkel EP, et al. Metagenomic shotgun sequencing and unbiased metabolomic profiling identify specific human gut microbiota and metabolites associated with immune checkpoint therapy efficacy in melanoma patients. Neoplasia. 2017;19:848-55.

36. Griffiths WJ, Koal T, Wang Y, Kohl M, Enot DP, Deigner H-P. Targeted metabolomics for biomarker discovery. Angew Chem Int Ed Engl. 2010;49:5426-45

37. Hale VL, Jeraldo P, Chen J, Mundy M, Yao J, Priya S, et al. Distinct microbes, metabolites, and ecologies define the microbiome in deficient and proficient mismatch repair colorectal cancers. Genome Med. 2018;10:78.

38. Nardi-Agmon I, Peled N. Exhaled breath analysis for the early detection of lung cancer: recent developments and future prospects. Lung Cancer. 2017;8:31-8.

39. Antoniou S, Gaude E, Ruparel M, van der Schee MP, Janes SM, Rintoul RC. The potential of breath analysis to improve outcome for patients with lung cancer. J Breath Res. 2019;13:034002.

40. Dent AG, Sutedja TG, Zimmerman PV. Exhaled breath analysis for lung cancer. J Thorac Dis. 2013;5(Suppl 5):S540-50.

41. Matsumura K, Opiekun M, Oka H, Vachani A, Albelda SM, Yamazaki K, et al. Urinary volatile compounds as biomarkers for lung cancer: a proof of principle study using odor signatures in mouse models of lung cancer. PLOS ONE. 2010;5:e8819.

42. Hanai Y, Shimono K, Matsumura K, Vachani A, Albelda S, Yamazaki K, et al. Urinary volatile compounds as biomarkers for lung cancer. Biosci Biotechnol Biochem. 2012;76:679-84.

43. El Hage R, Hernandez-Sanabria E, Calatayud Arroyo M, Props R, Van de Wiele T. Propionate-producing consortium restores antibiotic-induced dysbiosis in a dynamic in vitro model of the human intestinal microbial ecosystem. Front Microbiol. 2019;10:1206.

44. Louis P, Flint HJ. Formation of propionate and butyrate by the human colonic microbiota. Environ Microbiol. 2017;19:29-41.

45. Kim $\mathrm{CH}$. Immune regulation by microbiome metabolites. Immunology. 2018;154:220-9.

46. Heerdt BG, Houston MA, Augenlicht LH. Potentiation by specific shortchain fatty acids of differentiation and apoptosis in human colonic carcinoma cell lines. Cancer Res. 1994:54:3288-93.

47. Chen H-M, Yu Y-N, Wang J-L, Lin Y-W, Kong X, Yang C-Q, et al. Decreased dietary fiber intake and structural alteration of gut microbiota in patients with advanced colorectal adenoma. Am J Clin Nutr. 2013;97:1044-52.

48. Huda-Faujan N, Abdulamir AS, Fatimah AB, Anas OM, Shuhaimi M, Yazid $A M$, et al. The impact of the level of the intestinal short chain fatty acids in inflammatory bowel disease patients versus healthy subjects. Open Biochem J. 2010;4:53-8.

49. Bingula R, Filaire M, Radosevic-Robin N, Bey M, Berthon J-Y, BernalierDonadille A, et al. Desired turbulence? Gut-lung axis, immunity, and lung cancer. J Oncol. 2017;2017:5035371.

50. Pang T, Leach ST, Katz T, Day AS, Ooi CY. Fecal biomarkers of intestinal health and disease in children. Front Pediatr. 2014;2:6.

51. Saito Y, Sato T, Nomoto K, Tsuji H. Identification of phenol- and p-cresolproducing intestinal bacteria by using media supplemented with tyrosine and its metabolites. FEMS Microbiol Ecol. 2018;94:fiy125.

52. Andriamihaja M, Lan A, Beaumont M, Audebert M, Wong X, Yamada K, et al. The deleterious metabolic and genotoxic effects of the bacterial metabolite $\mathrm{p}$-cresol on colonic epithelial cells. Free Radic Biol Med. 2015;85:219-27.

53. Verbeke KA, Boobis AR, Chiodini A, Edwards CA, Franck A, Kleerebezem M, et al. Towards microbial fermentation metabolites as markers for health benefits of prebiotics. Nutr Res Rev. 2015:28:42-66.

54. Fangmann D, Theismann E-M, Türk K, Schulte DM, Relling I, Hartmann K, et al. Targeted microbiome intervention by microencapsulated delayedrelease niacin beneficially affects insulin sensitivity in humans. Diabetes Care 2018:41:398-405.

55. Bogan KL, Brenner C. Nicotinic acid, nicotinamide, and nicotinamide riboside: a molecular evaluation of NAD+ precursor vitamins in human nutrition. Annu Rev Nutr. 2008;28:115-30.

56. Nicholson JK, Lindon JC, Holmes E. "Metabonomics": understanding the metabolic responses of living systems to pathophysiological stimuli via multivariate statistical analysis of biological NMR spectroscopic data. Xenobiotica. 1999:29:1181-9.

57. Lindon JC, Nicholson JK. The emergent role of metabolic phenotyping in dynamic patient stratification. Expert Opin Drug Metab Toxicol. 2014;10:915-9.

\section{Publisher's Note}

Springer Nature remains neutral with regard to jurisdictional claims in published maps and institutional affiliations. 